\title{
Hypoxia promotes vasculogenic mimicry formation by the Twist1-Bmil connection in hepatocellular carcinoma
}

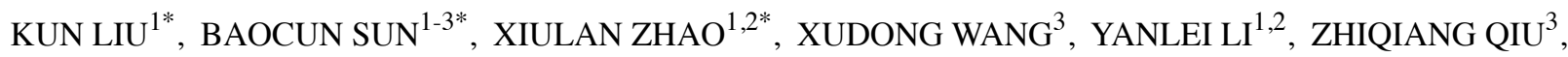 \\ TIEJU LIU ${ }^{1,2}$, QIANG GU ${ }^{1,2}$, XUEYI DONG ${ }^{1,2}$, YANHUI ZHANG ${ }^{3}$, YONG WANG ${ }^{1}$ and NAN ZHAO ${ }^{1}$ \\ ${ }^{1}$ Department of Pathology, Tianjin Medical University, Tianjin 300070; ${ }^{2}$ Department of Pathology, \\ Tianjin General Hospital, Tianjin Medical University, Tianjin 300052; ${ }^{3}$ Department of Pathology, \\ Tianjin Cancer Hospital, Tianjin Medical University, Tianjin 300060, P.R. China
}

Received May 7, 2015; Accepted June 29, 2015

DOI: $10.3892 /$ ijmm.2015.2293

\begin{abstract}
Aggressive tumor cells can mimic embryonic vasculogenic networks and form vasculogenic mimicry (VM). Preliminary studies demonstrated that hypoxia can promote VM formation; however, the underlying mechanism remains unclear. The present study aimed to investigate the role of the Twist1-Bmil connection in hypoxia-induced VM formation and the underlying mechanism. In the in vitro experiments, western blot analysis demonstrated that hypoxia upregulated the expression of Twist1, Bmi1, epithelial-mesenchymal transition (EMT) markers, stem cell markers and VM-associated markers. The 3D culture assay showed that hypoxia promoted VM formation in hepatocellular carcinoma (HCC) cell lines. Using transfection and in vitro cell experiments, the Twist1-Bmil connection was confirmed to have an important role in inducing EMT, cell stemness and VM formation. In the in vivo experiments, the murine hypoxia models were established via incomplete femoral artery ligation and the mechanism by which hypoxia promoted Twist1 and Bmil expression and led to VM formation was demonstrated by immunohistochemistry staining and endomucin/periodic acid Schiff double-staining. In conclusion, hypoxia upregulate the expression of Twist1 and Bmi1, and these two proteins have
\end{abstract}

Correspondence to: Professor Baocun Sun, Department of Pathology, Tianjin Medical University, No. 22 Qixiangtai Road, Heping, Tianjin 300070, P.R. China

E-mail: baocunsun@gmail.com

*Contributed equally

Abbreviations: $\mathrm{CoCl}_{2}$, cobalt chloride; CSCs, cancer stem cells; DMEM, Dulbecco's modified Eagle's medium; EMT, epithelial-mesenchymal transition; HCC, hepatocellular carcinoma; IHC, immunohistochemistry; MR, migration rate; PAS, periodic acid Schiff; VE-cadherin, vascular endothelial-cadherin; VM, vasculogenic mimicry

Key words: hypoxia, Twist1, Bmil, epithelial-mesenchymal transition, stemness, vasculogenic mimicry an important role in inducing EMT and cancer cell stemness, which contributed to VM formation.

\section{Introduction}

Hepatocellular carcinoma ( $\mathrm{HCC}$ ) is a prevalent malignancy and the most common malignant tumor of the liver. It requires an adequate blood supply to support its growth in vivo. Vasculogenic mimicry (VM) is an alternative pathway for the blood supply. VM is formed by tumor cells that mimic endothelial cells to form cell extracellular matrix-rich channels [periodic acid Schiff (PAS)-positive], which are sinusoidal structures that surround clusters of tumor cells. Studies have demonstrated that tumors, including HCC, may develop alternative pathways, such as VM and mosaic vessels $(1,2)$.

Hypoxia has an important role in various functional responses in tumor cells, including apoptosis and angiogenesis $(3,4)$. A previous study has demonstrated that hypoxia is critical in VM formation (5). Hypoxia can directly upregulate the expression of HIF-1 (SETD2), and HIF-1 can further combine to the promoter of Twist1 and promote its transcript expression. Twist1 is a basic helix-loop-helix transcription factor that can form homo- or hetero-dimers and bind the Ndel E-box element and activate or repress its target genes. Previous evidence has revealed the important role of Twistl in cancer metastasis, as shown by its overexpression in human cancers, induction of epithelial-mesenchymal transition (EMT) and association with a more malignant phenotype (6).

Previous studies identified that Bmil is directly regulated by the EMT regulator Twist1 (7). Bmil is a polycomb group family member that maintains self-renewal and is frequently overexpressed in human cancers (8). Twist1 and Bmil are mutually essential in promoting cell stemness, and the co-overexpression of Twist1 and Bmil can promote the tumor-initiating capability of cancer cells (7). This functional connection between Twist1 and Bmil provides a novel insight into the common mechanism mediating EMT and cancer stemness.

Although hypoxia has been reported to promote VM (5), the underlying mechanism remains unclear. In the present study, we hypothesized that hypoxia may lead to Twistl and Bmil upregulation, and the interaction between Twist1 and 
Bmil may promote VM formation by inducing EMT and cell stemness.

\section{Materials and methods}

Plasmid. The plasmid pEGFP-Twist1 was as previously described (9). The HepG2 cells were transfected with pEGFP-Twist1 or pcDNA control vector (pcDNA-negative).

Small interfering RNAs (siRNAs) encoding oligos against human Twist1 were as previously described (9). A non-silencing small interfering RNA sequence (target sequence, AATTCT CCGAACGTGTCACGT) was used as the negative control, as previously described (10). siRNA was used to block the Twist1 expression in Bel7402 cells.

Western blot analysis. Cells were washed using phosphate-buffered saline (PBS), and 10\% sodium dodecyl sulfate (SDS) was used to lyse the cells. The whole cell lysates were resolved via SDS-polyacrylamide gel electrophoresis and transferred onto polyvinylidene fluoride membranes (Millipore, Temecula, CA, USA). The membranes were blocked with skimmed milk powder and incubated with primary antibodies, followed by incubation with a secondary antibody, goat anti-rabbit (ZF-2301), goat anti-mouse (ZF-2305) or rabbit anti-goat (ZF-2305) immunoglobulin G-horseradish peroxidase (1:2,000; Zhongshan Chemical Co., Beijing, China). Protein expression was measured using an enhanced chemiluminescence detection kit (Amersham Pharmacia Biotech, Piscataway, NJ, USA). The primary antibodies for Twist1 (SC-15393; 1:200), E-cadherin (SC-7870; 1:200), Oct4 (SC-8629; 1:200) and cluster of differentiation 44 (CD44; SC-53298; 1:200) were purchased from Santa Cruz Biotechnology, Inc. (Dallas, TX, USA), Bmi1 (Ab14389; 1:1,000), vimentin (42011; 1:500; Epitomics), vascular endothelial-cadherin (VE-cadherin; AB-33168; 1:200) and $\beta$-actin (ab8226; 1:2,000) were purchased from Abcam (Cambridge, MA, USA), and fms-related tyrosine kinase 1 (FLT1; RB-1527; 1:200) and kinase insert domain receptor (KDR; RB-1526; 1:200) were purchased from Thermo Fisher Scientific (Waltham, MA, USA).

Cell culture and hypoxia treatment in vitro. Human liver cell lines HepG2 and Bel7402 were purchased from the American Type Culture Collection (Manassas, VA, USA). These cells were cultured in Dulbecco's modified Eagle's medium (DMEM) supplemented with $10 \%$ fetal bovine serum (FBS; Invitrogen, Carlsbad, CA, USA). Cobalt chloride $\left(\mathrm{CoCl}_{2}\right)$ was used to simulate hypoxic conditions. Cells were seeded in dishes or plates at 300 cells $/ \mathrm{mm}^{2}$ and grew for $24 \mathrm{~h}$ in complete medium. The medium was removed, and the cells were washed with PBS. The cells were treated with $100 \mu \mathrm{M} \mathrm{CoCl}_{2}$ and were subsequently incubated for different times as required.

$3 D$ culture assay. VM formation in vitro was evaluated using $3 \mathrm{D}$ culture. In the 3D culture assay, Matrigel (BD Biosciences, San Jose, CA, USA) was thawed at $4^{\circ} \mathrm{C}$ and $30 \mu \mathrm{l}$ was quickly added to each well of a 96-well plate and allowed to solidify for $12 \mathrm{~h}$ at $37^{\circ} \mathrm{C}$ in a humidified $5 \% \mathrm{CO}_{2}$ incubator. Tumor cells in complete medium were subsequently seeded onto the gel and incubated at $37^{\circ} \mathrm{C}$ for $24 \mathrm{~h}$. The formation of capillary-like structures was observed under a phase-contrast microscope (magnification, x200). Each experiment was performed in triplicate.

Wound-healing assay. In wound-healing assays, different groups of cells were plated in 24-well culture plates and allowed to grow for $24 \mathrm{~h}$. A micropipette was used to create a straight scratch in the center of each well. Cell migration ability was assessed by measuring the movement of cells into the scratch. The migration rate (MR) was monitored after 12, 24, 36 and $48 \mathrm{~h}$. The following formula was used to calculate the MR at different time-points: $M R=\left(d-d^{\prime}\right) / d(d$ is the length of the wound at $0 \mathrm{~h}$, and $\mathrm{d}^{\prime}$ is the length at other different time-points).

Invasion assay. In the invasion assay, $1 \times 10^{5}$ cells in $100 \mu 1$ of DMEM without FBS were seeded in the upper 24 wells coated with Matrigel matrix ( $1 \mathrm{mg} / \mathrm{ml}$; BD Biosciences) containing polyethylene terephthalate filters with $8-\mu \mathrm{m}$ porosity (Invitrogen). The lower chamber was filled with medium containing $10 \%$ FBS. The cells were incubated for $48 \mathrm{~h}$ and the non-invading cells on the upper surface of the membrane were removed. The cells that invaded through the Matrigel matrix and attached to the bottom surface of the membrane were fixed by methanol and stained by $0.5 \%$ crystal violet. The number of invading cells was counted using an inverted light microscope (Nikon, Tokyo, Japan).

Sphere culture assay. The sphere culture method was as described previously (11). Briefly, single HCC cells were seeded in 12-well plates coated with poly(2-hydroxyethylmethacrylate) (poly-HEMA; Sigma-Aldrich, St. Louis, MO, USA) at a density of $1 \times 10^{4}$ viable cells $/ \mathrm{ml}$ in a serum-free medium (DMEM-F12 1: 1 media; Gibco, Grand Island, NY, USA) supplemented with 1xB27 (Invitrogen), $10 \mathrm{ng} / \mathrm{ml}$ epidermal growth factor and $20 \mathrm{ng} / \mathrm{ml}$ fibroblast growth factor 2 (both from Peprotech, Rocky Hill, NY, USA), and 1\% pen/strep (Invitrogen). The culture medium was replaced or supplemented with additional growth factors every 3 days.

Clone formation assay. In the clone formation assay, each well of a 6 -well plate was seeded with 1,000 cells. The plates were incubated at $37^{\circ} \mathrm{C}$ and $5 \% \mathrm{CO}_{2}$ for 12 days, fixed with methanol and stained with $0.5 \%$ crystal violet. The number of clones was counted under the microscope.

Murine xenograft model. A total of 40 five-week-old male BALB/c nude mice were purchased from HuaFukang Biological Technology Co., Ltd, Beijing, China. All the studies on mice were carried out in accordance with the Guidelines of the Laboratory Animal of Tianjin Medical University (Tianjin, China). The mice were divided into two groups; the HepG2 and Bel7402 groups. All the animals received anesthesia with $2 \%$ pentobarbital sodium $(60 \mathrm{mg} / \mathrm{kg})$ prior to surgery. The overall surgical mortality rate was $0 \%$. Through a $1-1.5-\mathrm{cm}$ incision in the right groin, the femoral artery was carefully separated from the vein and nerve fiber, and ligated by $8-0$ silk with a needle placed along the vessel during ligation. The needles were $0.25 \mathrm{~mm}$ in diameter and were removed following ligation. The left limb was opened without femoral artery ligation as a control. Subsequently, $10^{7}$ cells suspended in $0.1 \mathrm{ml}$ of PBS were injected intramuscularly into both sides. The mice were monitored, and tumor sizes were measured daily using a Vernier caliper. The 
A

Twist1
Bmil
E-cadherin
Vimentin
CD44
Oct4
VE-cadherin
FLT1
KDR
$\beta$-actin

B

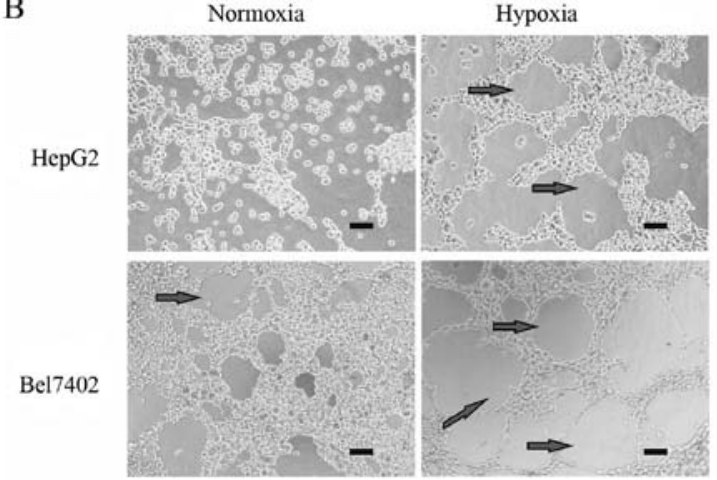

$\begin{array}{llllll}0 \mathrm{~h} & 12 \mathrm{~h} & 24 \mathrm{~h} & 36 \mathrm{~h} & 48 \mathrm{~h} & 60 \mathrm{~h}\end{array}$
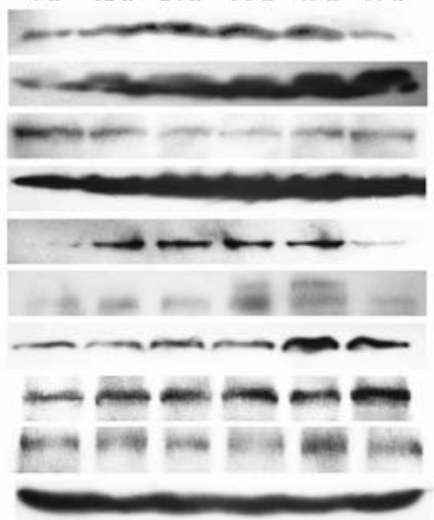

HepG2

Hypoxia

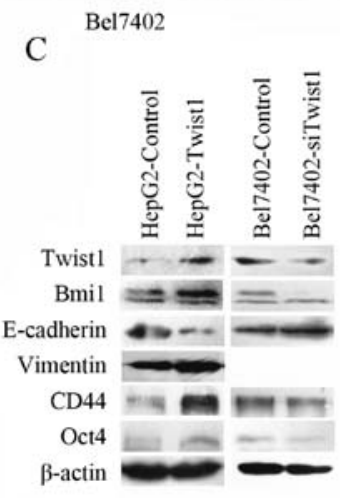

$\begin{array}{llllll}0 \mathrm{~h} & 12 \mathrm{~h} & 24 \mathrm{~h} & 36 \mathrm{~h} & 48 \mathrm{~h} & 60 \mathrm{~h}\end{array}$
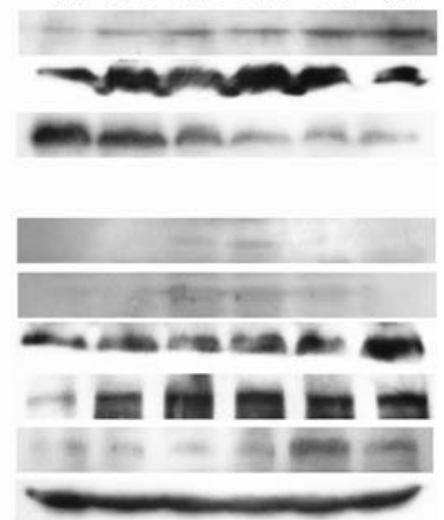

Figure 1. Effects of hypoxia and transfection on hepatocellular carcinoma cell lines. (A) Protein expression levels of Twist1, Bmil, stem cell, EMT and VM-associated markers in HepG2 and Bel7402 cells after different incubation times of hypoxia (0, 12, 24, 36, 48 and 60 h). $\beta$-actin was used as loading control. The two groups demonstrated a significant increase in Twistl and Bmil expression with time under hypoxia. The expression of E-cadherin in the two cell lines was downregulated and the expression of vimentin in HepG2 was upregulated by hypoxia, indicative of EMT. Vimentin expression was not observed in Bel7402 cells. The expression of stem cell markers and VM-associated markers was also promoted by hypoxia. (B) Effect of hypoxia on VM formation in HCC cells cultured on Matrigel matrix (magnification, x100; bars, $100 \mu \mathrm{m}$ ). HepG2 cells did not form typical tube-like structures, whereas Bel7402 cells formed a modest level of tube formation. After hypoxia was administered, VM formation was significantly induced. The arrows show the tube-like structures in the 3D culture. (C) Western blot results showed that the Bmil expression level was upregulated following Twist1 transfection and downregulated following siTwist1 transfection. The results also revealed that vimentin expression was increased and E-cadherin expression was decreased in the HepG2 cells following Twist1/Bmi1 upregulation. By contrast, E-cadherin expression was increased in Bel7402 cells following Twist1/Bmi1 downregulation. The stem cell marker CD44 and Oct4 expression was also upregulated by Twist1/Bmi1 upregulation and downregulated by Twist1/Bmi1 downregulation. CD44, cluster of differentiation 44; EMT, epithelial-mesenchymal transition; HCC, hepatocellular carcinoma; VM, vasculogenic mimicry.

following formula was used to calculate tumor volume: Tumor volume $=1 / 2 \mathrm{ab}^{2}$ ( $\mathrm{a}$ is the length and $\mathrm{b}$ is the width of the tumor). When the observation was complete, the mice were sacrificed. Tumors were harvested and fixed by formalin.

Immunohistochemistry (IHC) and endomucin/PAS double-staining. Prior to immunostaining, $4-\mu \mathrm{m}$ paraffin sections were deparaffinized in xylene and rehydrated by a graded series of aqueous ethanol solutions. Endogenous peroxidase activity was blocked with $3 \%$ hydrogen peroxide in $100 \%$ methanol for $30 \mathrm{~min}$ at room temperature. The sections were pretreated in a microwave, blocked and incubated using a series of antibodies. The staining systems used in the present study were PicTure PV6000 (Zhongshan Chemical Co.). In endomucin/PAS double-staining, the sections were washed with running water for $5 \mathrm{~min}$ and were subsequently incubated with PAS for 15 min after IHC for endomucin was performed. Finally, the sections were counterstained with hematoxylin, dehydrated and mounted. PBS was used instead of the primary antibodies for the negative control. The results were quantified according to the method described by Bittner et al (12).
Statistical analysis. All the data in the study were evaluated with SPSS 17.0 software (SPSS Inc., Chicago, IL, USA). Student's t-test was performed to determine differences between two groups in cell functional assays. Paired t-test was performed to compare the hypoxia and control groups in vivo. $\mathrm{P}<0.05$ was considered to indicate a statistically significant difference.

\section{Results}

\section{Effect of hypoxia in vitro}

Hypoxia results in Twist1-Bmil upregulation. HepG2 and Bel7402 cells were used in the study to verify the association of hypoxia with VM in HCC cells in vitro. HepG2 and Bel7402 cells were cultured under hypoxic conditions, and Twist1 and Bmil protein expression levels were detected by western blot analysis after $0,12,24,36,48$ or $60 \mathrm{~h}$ of hypoxia. The two groups demonstrated a significant increase in Twist1 and Bmi1 expression with time under hypoxia (Fig. 1A).

Hypoxia induces EMT and cell stemness. Accompanied with Twist1/Bmi1 upregulation, the expression of E-cadherin 
A

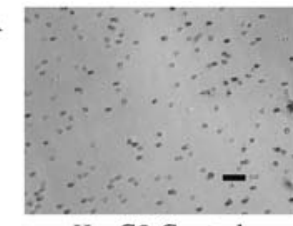

HepG2-Control

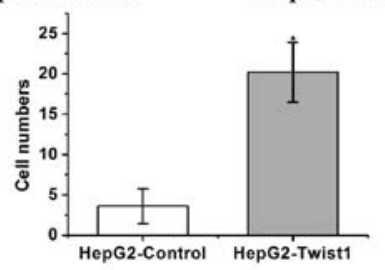

B

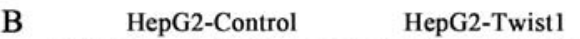

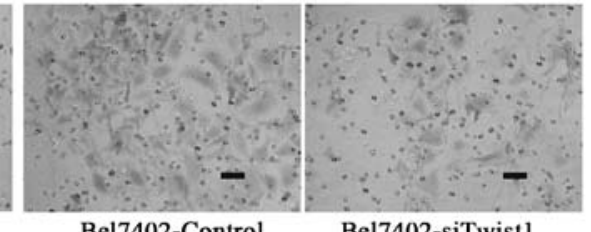

Bel7402-Control

Bel7402-siTwistl

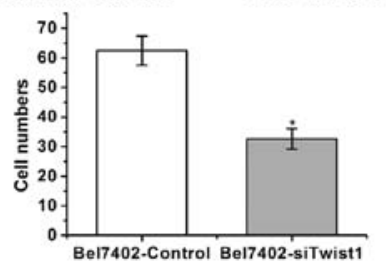

Bel7402-Control

Bel7402-siTwist1

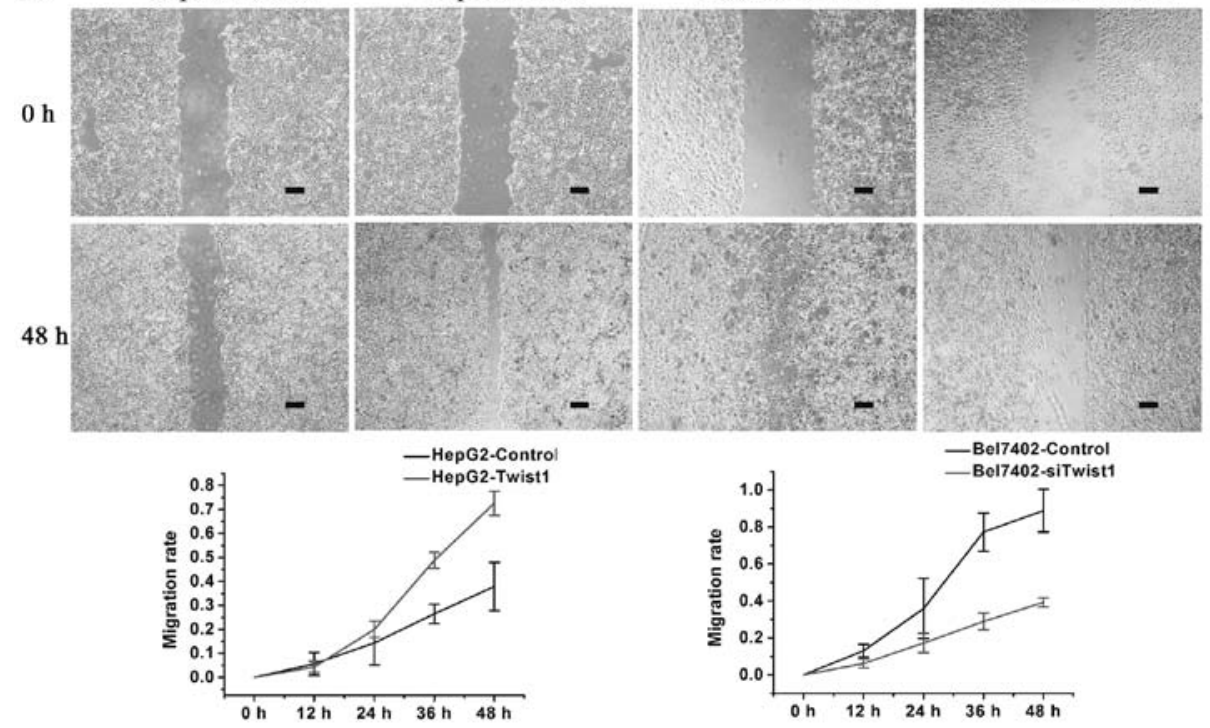

Figure 2. Changes in the capacity of invasion and metastasis of the transfected cells. (A) Number of invasion cells was assessed in the invasion assay (magnification, x200; bars, $50 \mu \mathrm{m}$ ). Results showed that Twist1 upregulation enhanced HepG2 cell invasion and Twist1 downregulation inhibited Bel7402 cell invasion $\left({ }^{*} \mathrm{P}<0.05\right)$. (B) Wound-healing assays (magnification, $\mathrm{x} 40$; bars, $200 \mu \mathrm{m}$ ) revealed that HepG2-Twist1 cells exhibited a higher motility compared with the HepG2-control cells. Bel7402-control cells exhibited a higher motility compared with the Bel7402-siTwist1 cells. The results were statistically significant $(\mathrm{P}<0.05)$.

in the two cells lines was downregulated and the expression of vimentin in HepG2 was upregulated by hypoxia, indicative of EMT (Bel7402 cells did not show any vimentin expression; Fig. 1A). The expression of stem cell markers was also promoted by hypoxia. Stem cell marker Oct4 (OCT3/4, POU5F1) is a member of the POU transcription factor family, and it has a critical role in maintaining cell stemness (13). CD44 is a major extracellular matrix adhesion molecule that is widely used as a cancer stem cell marker in various types of cancer, including HCC $(14,15)$. Western blot analysis showed that hypoxia increased the expression of CD44 and Oct4 in HepG2 and Bel7402 cells (Fig. 1A).

Hypoxia promotes VM formation ability of HCC cells. The expression of VM-associated markers VE-cadherin, FLT1 and KDR were also elevated by hypoxia (Fig. 1A). The ability of VM formation on Matrigel of HepG2 and Bel7402 cells under normoxia and cells that underwent $48 \mathrm{~h}$ of hypoxia was assessed. Hypoxia promoted VM formation in the HepG2 and Bel7402 cells (Fig. 1B).

Twist1-Bmil cooperation is a critical factor in VM formation Bmil expression is regulated by Twist1. The endogenous expression of Twist 1 in the HepG2 cell line was significantly lower compared with the Bel7402 cell line. Thus, HepG2 cells were infected with Twist1 plasmid and the Bel7402 cells with siTwist1 plasmid. Western blot analysis showed that Bmil expression was upregulated in HepG2-Twist1 cells and downregulated in Bel7402-siTwist1 cells (Fig. 1C), confirming that Bmil expression was regulated by Twist1.

Upregulation of Twistl and Bmil induces EMT and cell stemness. Western blot analysis showed that in HepG2 cells, the upregulation of Twistl promoted vimentin expression and inhibited E-cadherin expression, indicative of EMT. In Bel7402 cells, the downregulation of Twist1 promoted E-cadherin expression. The expression levels of stem cell marker CD44 and Oct4 were upregulated in HepG2-Twist1 cells and downregulated in Bel7402-siTwist1 cells (Fig. 1C).

The tumor cells that exhibited the ability to undergo EMT also had a greater ability to invade and metastasize. The HepG2-control, HepG2-Twist1, Bel7402-control and Bel7402-siTwist1 cells were subsequently analyzed for functional changes in invasion and migration ability. The in vitro invasion assay demonstrated that upregulation of Twist1 in HepG2 cells significantly promoted cell invasion, while downregulation of Twist1 in Bel7402 cells significantly inhibited cell invasion (Fig. 2A). In the wound-healing assay, the MR at 
A

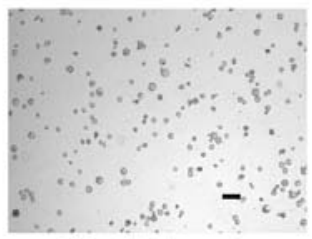

HepG2-Control

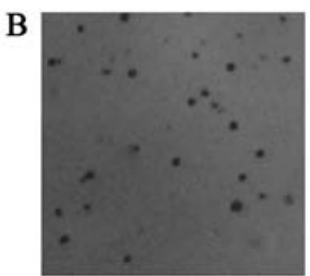

HepG2-Control

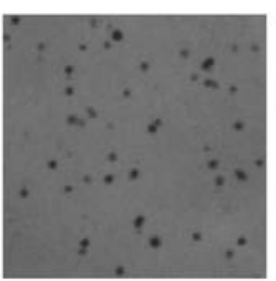

HepG2-Twist1

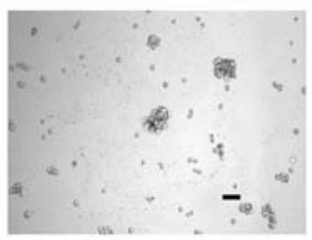

HepG2-Twist1

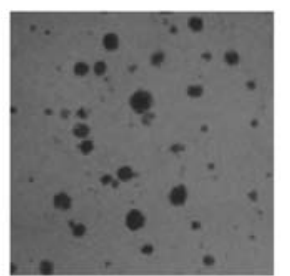

Bel7402-Control

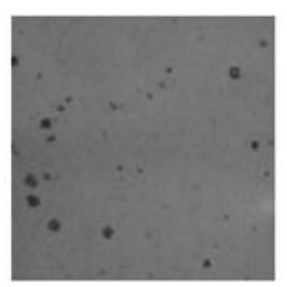

Bel7402-siTwist1
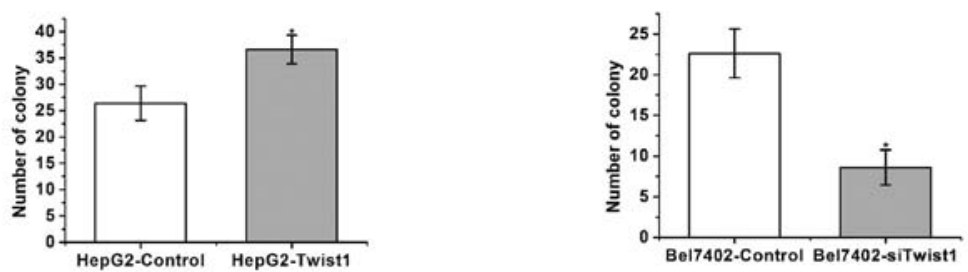

C

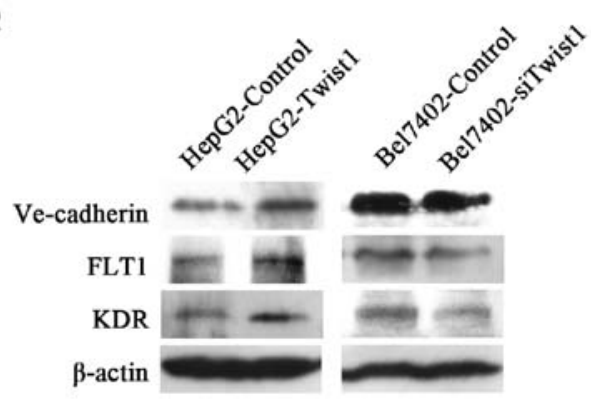

D

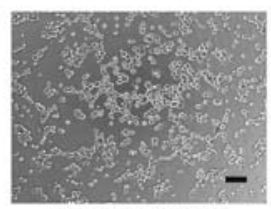

HepG2-Control

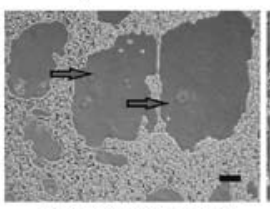

Bel7402-Control

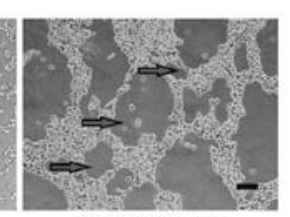

HepG2-Twist1

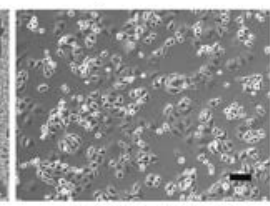

Bel7402-siTwist1

Figure 3. Changes in cell stemness and vasculogenic mimicry formation ability in transfected cells. (A) Viable sphere formation was observed in the HepG2-Twist1 group, however, no spheres were formed in the HepG2-control group (magnification, x100; bars, $100 \mu \mathrm{m}$ ). (B) Clonogenic assay showed increased cell colony formation ability in the HepG2-Twist1 group and decreased cell colony formation ability in the Bel7402-siTwist1 group compared with the control groups ( $\left.{ }^{*} \mathrm{P}<0.05\right)$. (C) Western blot results showed that the upregulation of Twist1 promoted the expression of VM-associated markers. By contrast, downregulation of Twist1 inhibited the expression of VM-associated markers. (D) Bel7402-control cells exhibited a higher tube formation capacity compared with the HepG2-control cells in the 3D culture experiments. Twist1 enhanced the tube formation capacity of HepG2 cells, and siTwist1 weakened this capacity in the Bel7402 cells (magnification, x100; bars, $100 \mu \mathrm{m}$ ). VM, vasculogenic mimicry.

the terminal phase ( $48 \mathrm{~h}$ ) increased in the HepG2-Twist1 group and decreased in the Bel7402-siTwistl group compared with the control groups (Fig. 2B).

Sphere culture and clone formation assays were performed to evaluate cell stemness. In the sphere culture assay, HepG2-control, HepG2-Twist1, Bel7402-control and Bel7402-siTwist1 cells were seeded in plates coated with poly-HEMA at a density of $1 \times 10^{4}$ cells $/ \mathrm{ml}$ using a commonly used suspension culture containing $1 \%$ methylcellulose. After 4-7 days of culture, a viable sphere formation was observed in the HepG2-Twistl group, however, no spheres were formed in the HepG2-control group (Fig. 3A). Neither Bel7402-control nor Bel7402-siTwistl group formed spheres (data not shown). In the clone formation assay, Twist1 upregulation in HepG2 cells resulted in the significant promotion of cell colony formation, while in Bel7402 cells, Twist1 downregulation significantly inhibited colony formation (Fig. 3B).
Expression levels of Twistl and Bmil influences VM formation. Western blot analysis showed that the expression levels of VM-associated markers, VE-cadherin, FLT1 and $\mathrm{KDR}$, were upregulated in HepG2-Twist1 cells and downregulated in Bel7402-siTwist1 cells (Fig. 3C). The formation of capillary-like structures was assessed by the 3D culture assay. HepG2-control cells could not form any tube-like structures, while HepG2-Twist1 formed typical tube-like structures on the surface of the Matrigel medium. Bel7402-control cells showed modest tube-like structures while Bel7402-siTwist1 cells showed no tube-like structures (Fig. 3D).

\section{Effect of hypoxia in vivo}

Hypoxia promotes Twistl and Bmil expression and induces EMT and cell stemness in vivo. An incomplete femoral artery ligation murine model was established to investigate the effects of hypoxia in vivo. A total of 14 mice carried tumors 


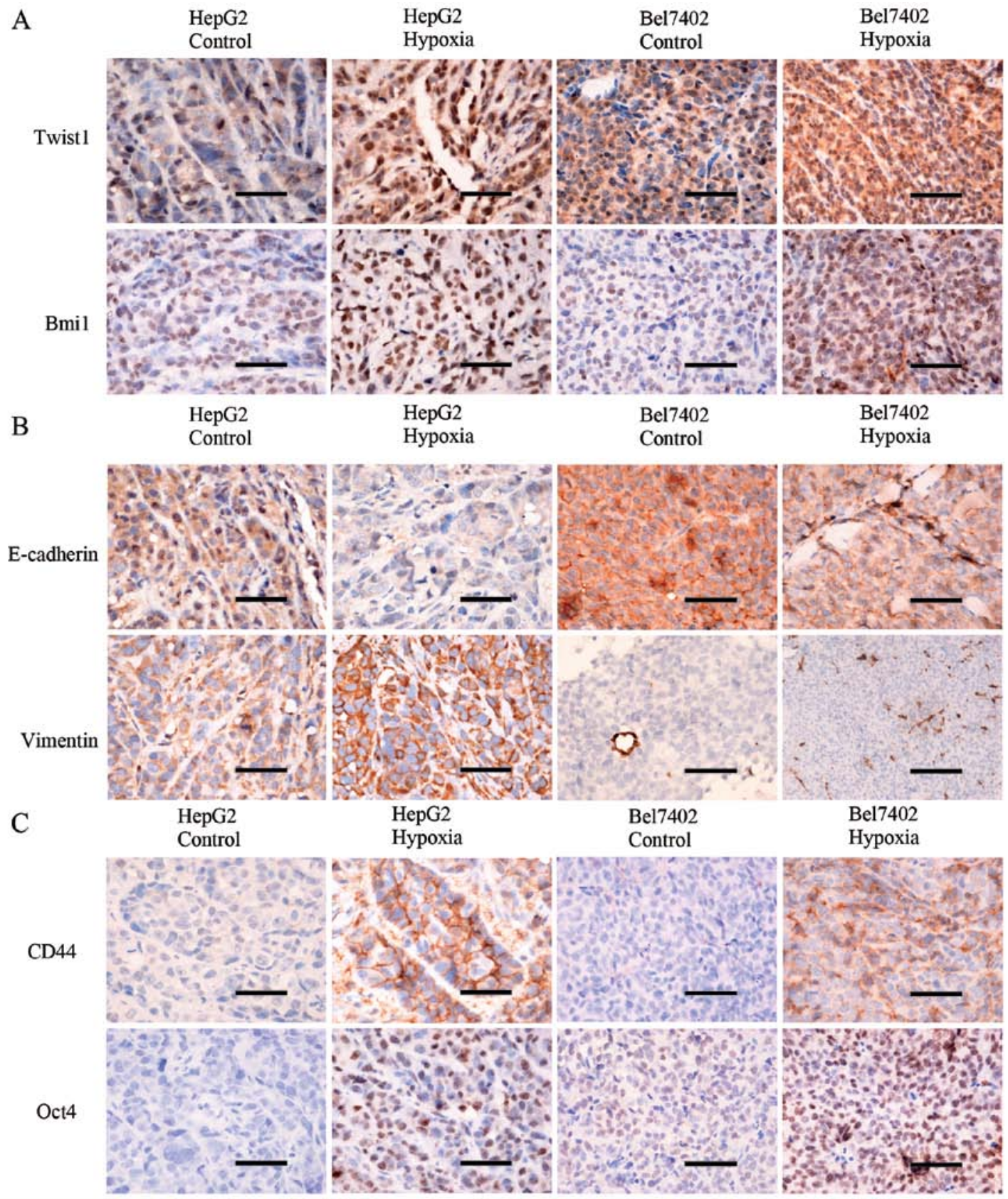

Figure 4. Hypoxia upregulates Twist1 and Bmil expression and promotes epithelial mesenchymal transition and stemness in vivo. (A) Expression levels of Twist1 and Bmi1 in HepG2- and Bel7402-engrafted tumors (magnification, x400; bars, $50 \mu \mathrm{m}$ ) were detected by IHC staining. Twist1 and Bmil expression levels were higher in the hypoxia group compared with those in the control group ( $\mathrm{P}<0.05)$. (B) Expression levels of EMT markers in HepG2-and Bel7402-engrafted tumors (magnification, $\mathrm{x} 400 ;$ bars, $50 \mu \mathrm{m}$ ). The HepG2 hypoxia and control groups exhibited positive staining for E-cadherin in the plasma membrane and vimentin in the cytoplasm. The Bel7402 control and hypoxia groups exhibited positive staining for E-cadherin in the plasma membrane but negative staining for vimentin in tumor cells. The expression of E-cadherin was downregulated and the expression of vimentin was upregulated in the hypoxia groups, but not in the Bel7402 groups $(\mathrm{P}<0.05)$. (C) IHC staining also showed that the expression levels of the stem cell markers was higher in the hypoxia group compared with that in the control group (magnification, $\mathrm{x} 400$; bars, $50 \mu \mathrm{m} ; \mathrm{P}<0.05$ ). IHC, immunohistochemistry; EMT, epithelial-mesenchymal transition.

in the bilateral hindlimbs in the HepG2 group and there were 7 mice in the Bel7402 groups. Using IHC, Twist1, Bmi1, EMT marker and stem cell marker expression was examined in the engrafted tumors in the HepG2 control, HepG2 hypoxia, Bel7402 control, and Bel7402 hypoxia groups. Cells exhibited positive staining for Twist1 in the nucleus and cytoplasm, Bmi1 in the nucleus, E-cadherin in the plasma membrane, vimentin in the plasma membrane and cytoplasm, CD44 in the plasma membrane, and Oct4 in the nucleus (Fig. 4). The expression of Twistl and Bmil expression in the hypoxia group was stronger compared with that in the control group ( $\mathrm{P}<0.05$; Fig. 4A). The expression of E-cadherin was downregulated and the expression of vimentin was upregulated in the hypoxia group (tumor cells in Bel7402-engrafted tumors showed no vimentin expression) $(\mathrm{P}<0.05$; Fig. 4B). The expression of stem cell marker CD44 and Oct4 was also stronger in the hypoxia group compared with that in the control group in the HepG2- and Bel7402-engrafted tumors ( $\mathrm{P}<0.05$; Fig. 4C).

Hypoxia promotes VM in vivo. The expression levels of VM-associated markers, including VE-cadherin, FLT1 and 

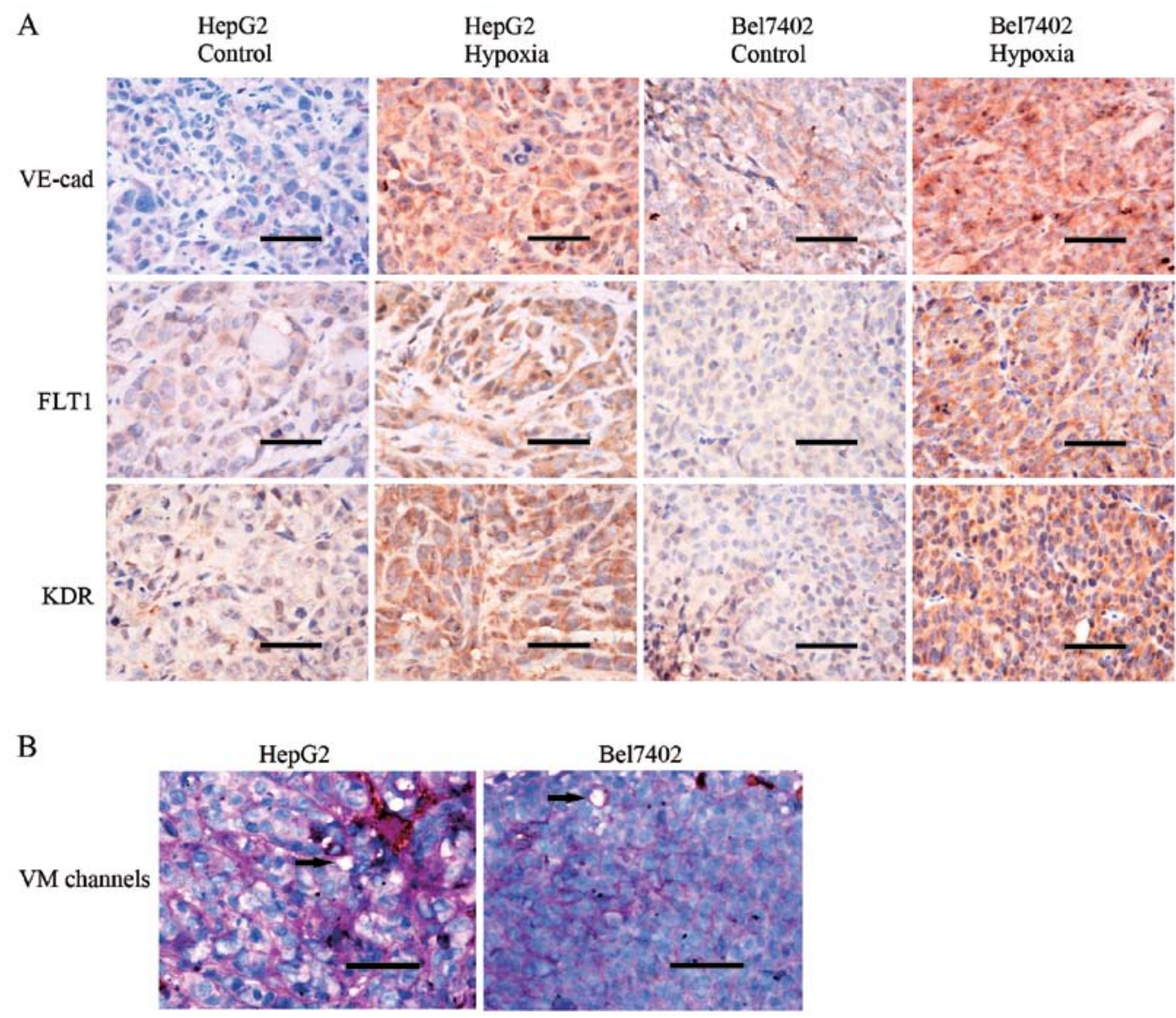

Figure 5. Hypoxia promotes vasculogenic mimicry formation in vivo. (A) Expression levels of VM-associated markers in HepG2- and Bel7402-engrafted tumors (magnification, $\mathrm{x} 400$; bars, $50 \mu \mathrm{m}$ ). The expression of the VM-associated markers was higher in the hypoxia group compared with the control group $(\mathrm{P}<0.05)$. (B) Arrows show VM channels in engrafted tumors. VM channels in HepG2- and Bel7402-engrafted tumors formed by tumor cells, which were negative for endomucin; the basement membrane-like structure that lined the channel was positive for PAS (magnification, $\mathrm{x} 400$; bars, $50 \mu \mathrm{m}$ ). VM, vasculogenic mimicry; PAS, periodic acid Schiff.

KDR, were examined using IHC staining (Fig. 5A). The control and hypoxia groups contained plasma membrane VE-cadherin-, cytoplasmic VEGFR1- and cytoplasmic VEGFR2-positive tumor cells. Tumors in the hypoxia group expressed more of these proteins compared with the control group $(\mathrm{P}<0.05)$. Using endomucin/PAS double-staining, VM channels (Fig. 5B) were observed in the engrafted tumors. VM channels were formed by tumor cells lined with PAS-positive substances, and these cells were negative for endomucin. Hypoxia promoted VM formation. The HepG2 hypoxia group had $5.14 \pm 1.505 \mathrm{VM}$ channels, whereas the control group had $3.00 \pm 0.756 \mathrm{VM}$ channels $(\mathrm{P}<0.05)$. The Bel7402 hypoxia group had 3.29 $\pm 0.700 \mathrm{VM}$ channels, whereas the control group had $1.43 \pm 0.495 \mathrm{VM}$ channels $(\mathrm{P}<0.05)$.

\section{Discussion}

Hypoxia has a pivotal role in the microenvironment, and a hypoxic microenvironment could affect tumor angiogenesis, metastasis, metabolism and the survival of tumor cells $(5,16,17) \cdot \mathrm{CoCl}_{2}$ is a hypoxia-mimicking reagent that is commonly used in hypoxia studies in vitro $(18,19)$. It mimics hypoxic conditions by inhibiting the activity of prolyl hydroxylase, a critical enzyme in the oxygen-sensing pathway (20). In the present study, $\mathrm{CoCl}_{2}$ was used in cell cultures to simulate constant hypoxia. In the in vivo experiments, incomplete femoral artery ligation was performed in nude mice to create hypoxic conditions, as serious gangrene could occur following complete femoral artery ligation and excision in the hindlimb of nude mice (21).

Hypoxia has been proved to promote VM formation (5). VM was first reported in highly aggressive uveal melanoma in 1999 by Maniotis et al (22). VM has been found in HCC samples, and studies have reported that VM is associated with metastasis and a shorter survival period in HCC (23). Tumor cells that formed the unique structure of VM channels were directly exposed to the bloodstream, so they could easily metastasize to distant sites by entering the microcirculation. This discovery may explain the elevated risk of metastasis, tumor recurrence and shorter survival period in patients with VM-positive HCC. Hypoxia is a crucial factor in VM formation $(5,9)$.

The EMT regulator Twistl has been reported to regulate the expression of Bmil directly, which provides a novel insight into the association between EMT and cancer stemness (7). The Twist1-Bmil link was responsible for inducing EMT and cell stemness under hypoxia conditions. EMT is a pivotal developmental program that is often activated in cancer development $(24,25)$. A number of changes may occur to epithelial cells during EMT, including loss of epithelial characteristics, 
acquisition of mesenchymal phenotypes, and ability to invade, resist apoptosis and disseminate. Twist1 is an EMT regulator that regulates EMT in various tumors $(26,27)$, and can be upregulated by hypoxia. Twistl can repress E-cadherin and promote mesenchymal markers expression (28). Bmil is directly regulated by Twist1. Twist1 and Bmil are mutually essential in promoting cell stemness, and the co-overexpression of Twist1 and Bmil can promote the tumor-initiating capability of cancer cells (7). The present study also showed that the expression of CD44 and Oct4 was upregulated following Twist1-Bmil cooperation. Oct4 has an important role in maintaining stemness (29). CD44 is a major extracellular matrix adhesion molecule that is widely used as a cancer stem cell marker in various cancer types, including HCC $(14,15)$. Bmi1 has been reported to be co-expressed with CD44 in cancer stem cells (CSCs) $(30,31)$. Thus, the present study showed that cell stemness was induced by hypoxia. Stemness and EMT are interconnected processes. Twist1/Bmil-induced stemness may be an important section of the EMT progress. The epithelial cells may first obtain high levels of stemness, and subsequently, they can differentiate toward mesenchymal-like cells. Ectopic expression of Bmil in normal nasopharyngeal epithelial cells is sufficient to cause EMT (32). A recent breakthrough in metastasis studies demonstrated that induction of EMT also generates cells with stem-like properties $(33,34)$. The mutual promotion of these two processes is the basis of VM formation.

The present study demonstrated increased VM formation following the induction of EMT and cell stemness. EMT and stemness can induce VM formation. Twistl can induce EMT, and EMT can contribute to tumor cell plasticity; the general mechanism behind this phenomenon involves inhibiting E-cadherin transcription caused by Twist1 interacting with its promoter and further induction of the expression of downstream mesenchymal markers (6). This process is characteristic of VM formation, which involves tumor cells mimicking endothelial cells, a type of mesenchymal cell, similar to the EMT process. Tumor cell stemness is associated with VM formation; CSCs can undergo asymmetric cell division, which results in tumor cells that promote angiogenesis (35). CSCs may express an endothelial phenotype and form vessel-like networks, thereby mimicking the pattern of vascular networks in the embryo (36,37). Tumor cells expressing high stemness can exist in HCC, and this stem cell population is responsible for VM formation (38). The present study indicated that Twist1-Bmi1 cooperation induced VM formation through induction of EMT and tumor cell stemness. Increased expression of VM-associated markers and VM formation was observed following induced EMT and stemness in vitro and in vivo.

In conclusion, the present study demonstrated that the effects of hypoxia on VM formation in HCC cells involved the Twist1-Bmil connection, which induced EMT and stemness. Studies on VM mechanisms could help us develop appropriate drugs to inhibit tumor growth and metastasis by suppressing VM. Inhibitors could be specially designed to inhibit Twist1, Bmil or their associated markers.

\section{Acknowledgements}

The present study was supported by grants from the Key Project of the National Natural Science Foundation of China (no. 81230050), the National Natural Science Foundation of China (nos. 81172046, 81173091 and 81201791), the Key Project of the Tianjin Natural Science Foundation (no. 12JCZDJC23600), and the Natural Science Foundation of Tianjin Education Commission (no. 20120103).

\section{References}

1. Bai XL, Zhang Q, Ye LY, Liang F, Sun X, Chen Y, Hu QD, Fu QH, $\mathrm{Su} \mathrm{W}$, Chen Z, et al: Myocyte enhancer factor $2 \mathrm{C}$ regulation of hepatocellular carcinoma via vascular endothelial growth factor and Wnt/ $\beta$-catenin signaling. Oncogene: Oct 20, 2014 (Epub ahead of print). doi: 10.1038/onc.2014.337.

2. Deng JH and Li HZ: Vasculogenic mimicry and mosaic vessels and targeted therapy in renal cell carcinoma. Zhongguo Yi Xue Ke Xue Yuan Xue Bao 33: 462-467, 2011 (In Chinese).

3. Zhu P, Ning Y, Yao L, Chen M and Xu C: The proliferation, apoptosis, invasion of endothelial-like epithelial ovarian cancer cells induced by hypoxia. J Exp Clin Cancer Res 29: 124, 2010.

4. Fan L, Li J, Yu Z, Dang X and Wang K: Hypoxia-inducible factor prolyl hydroxylase inhibitor prevents steroid-associated osteonecrosis of the femoral head in rabbits by promoting angiogenesis and inhibiting apoptosis. PLoS One 9: e107774, 2014.

5. Du J, Sun B, Zhao X, Gu Q, Dong X, Mo J, Sun T, Wang J, Sun R and Liu Y: Hypoxia promotes vasculogenic mimicry formation by inducing epithelial-mesenchymal transition in ovarian carcinoma. Gynecol Oncol 133: 575-583, 2014.

6. Qin Q, Xu Y, He T, Qin C and Xu J: Normal and disease-related biological functions of Twist1 and underlying molecular mechanisms. Cell Res 22: 90-106, 2012.

7. Yang MH, Hsu DS, Wang HW, Wang HJ, Lan HY, Yang WH, Huang CH, Kao SY, Tzeng CH, Tai SK, et al: Bmil is essential in Twist1-induced epithelial-mesenchymal transition. Nat Cell Biol 12: 982-992, 2010.

8. Siddique HR and Saleem M: Role of BMI1, a stem cell factor, in cancer recurrence and chemoresistance: Preclinical and clinical evidences. Stem Cells 30: 372-378, 2012.

9. Sun T, Sun BC, Zhao XL, Zhao N, Dong XY, Che N, Yao Z, Ma YM, Gu Q, Zong WK, et al: Promotion of tumor cell metastasis and vasculogenic mimicry by way of transcription coactivation by Bcl-2 and Twist1: A study of hepatocellular carcinoma. Hepatology 54: 1690-1706, 2011.

10. Sun T, Zhao N, Zhao XL, Gu Q, Zhang SW, Che N, Wang XH, Du J, Liu YX and Sun BC: Expression and functional significance of Twist1 in hepatocellular carcinoma: Its role in vasculogenic mimicry. Hepatology 51: 545-556, 2010.

11. Wong CE, Paratore C, Dours-Zimmermann MT, Rochat A, Pietri T, Suter U, Zimmermann DR, Dufour S, Thiery JP, Meijer D, et al: Neural crest-derived cells with stem cell features can be traced back to multiple lineages in the adult skin. J Cell Biol 175: 1005-1015, 2006.

12. Bittner M, Meltzer P, Chen Y, Jiang Y, Seftor E, Hendrix M, Radmacher M, Simon R, Yakhini Z, Ben-Dor A, et al: Molecular classification of cutaneous malignant melanoma by gene expression profiling. Nature 406: 536-540, 2000.

13. Nichols J,Zevnik B, Anastassiadis K, Niwa H, Klewe-Nebenius D, Chambers I, Schöler H and Smith A: Formation of pluripotent stem cells in the mammalian embryo depends on the POU transcription factor Oct4. Cell 95: 379-391, 1998.

14. Angeloni V, Tiberio P, Appierto V and Daidone MG: Implications of stemness-related signaling pathways in breast cancer response to therapy. Semin Cancer Biol 31: 43-51, 2015.

15. Chen R, Dong Y, Xie X, Chen J, Gao D, Liu Y, Ren Z and Cui J: Screening candidate metastasis-associated genes in three-dimensional HCC spheroids with different metastasis potential. Int J Clin Exp Pathol 7: 2527-2535, 2014.

16. Wong CC, Kai AK and Ng IO: The impact of hypoxia in hepatocellular carcinoma metastasis. Front Med 8: 33-41, 2014.

17. Marie-Egyptienne DT, Lohse I and Hill RP: Cancer stem cells, the epithelial to mesenchymal transition (EMT) and radioresistance: Potential role of hypoxia. Cancer Lett 341: 63-72, 2013.

18. Bae S, Jeong HJ, Cha HJ, Kim K, Choi YM, An IS, Koh HJ, Lim DJ, Lee SJ and An S: The hypoxia-mimetic agent cobalt chloride induces cell cycle arrest and alters gene expression in U266 multiple myeloma cells. Int J Mol Med 30: 1180-1186, 2012. 
19. Bauer N, Liu L, Aleksandrowicz E and Herr I: Establishment of hypoxia induction in an in vivo animal replacement model for experimental evaluation of pancreatic cancer. Oncol Rep 32: 153-158, 2014.

20. Salnikow K, Donald SP, Bruick RK, Zhitkovich A, Phang JM and Kasprzak KS: Depletion of intracellular ascorbate by the carcinogenic metals nickel and cobalt results in the induction of hypoxic stress. J Biol Chem 279: 40337-40344, 2004.

21. Kang Y,Park C,Kim D, Seong CM, Kwon K and Choi C: Unsorted human adipose tissue-derived stem cells promote angiogenesis and myogenesis in murine ischemic hindlimb model. Microvasc Res 80: 310-316, 2010.

22. Maniotis AJ, Folberg R, Hess A, Seftor EA, Gardner LM, Pe'er J, Trent JM, Meltzer PS and Hendrix MJ: Vascular channel formation by human melanoma cells in vivo and in vitro: Vasculogenic mimicry. Am J Pathol 155: 739-752, 1999.

23. Sun B, Zhang S, Zhang D, Du J, Guo H, Zhao X, Zhang W and Hao X: Vasculogenic mimicry is associated with high tumor grade, invasion and metastasis, and short survival in patients with hepatocellular carcinoma. Oncol Rep 16: 693-698, 2006.

24. Thiery JP, Acloque H, Huang RY and Nieto MA: Epithelial-mesenchymal transitions in development and disease. Cell 139: 871-890, 2009.

25. Raimondi C, Gianni W, Cortesi E and Gazzaniga P: Cancer stem cells and epithelial-mesenchymal transition: Revisiting minimal residual disease. Curr Cancer Drug Targets 10: 496-508, 2010

26. Biroccio A, Candiloro A, Mottolese M, Sapora O, Albini A, Zupi G and Del Bufalo D: Bcl-2 overexpression and hypoxia synergistically act to modulate vascular endothelial growth factor expression and in vivo angiogenesis in a breast carcinoma line. FASEB J 14: 652-660, 2000.

27. Kang Y and Massagué J: Epithelial-mesenchymal transitions: Twist in development and metastasis. Cell 118: 277-279, 2004.

28. Thiery JP and Sleeman JP: Complex networks orchestrate epithelial-mesenchymal transitions. Nat Rev Mol Cell Biol 7: 131-142, 2006.
29. Koo BS, Lee SH, Kim JM, Huang S, Kim SH, Rho YS, Bae WJ, Kang HJ, Kim YS, Moon JH, et al: Oct4 is a critical regulator of stemness in head and neck squamous carcinoma cells. Oncogene 34: 2317-2324, 2015.

30. Prince ME, Sivanandan R, Kaczorowski A, Wolf GT, Kaplan MJ, Dalerba P, Weissman IL, Clarke MF and Ailles LE: Identification of a subpopulation of cells with cancer stem cell properties in head and neck squamous cell carcinoma. Proc Natl Acad Sci USA 104: 973-978, 2007.

31. Zhang S, Balch C, Chan MW, Lai HC, Matei D, Schilder JM, Yan PS, Huang TH and Nephew KP: Identification and characterization of ovarian cancer-initiating cells from primary human tumors. Cancer Res 68: 4311-4320, 2008.

32. Song LB, Li J, Liao WT, Feng Y, Yu CP, Hu LJ, Kong QL, Xu LH, Zhang X, Liu WL, et al: The polycomb group protein Bmi-1 represses the tumor suppressor PTEN and induces epithelial-mesenchymal transition in human nasopharyngeal epithelial cells. J Clin Invest 119: 3626-3636, 2009.

33. Mani SA, Guo W, Liao MJ, Eaton EN, Ayyanan A, Zhou AY, Brooks M, Reinhard F, Zhang CC, Shipitsin M, et al: The epithelial-mesenchymal transition generates cells with properties of stem cells. Cell 133: 704-715, 2008.

34. Morel AP, Lièvre M, Thomas C, Hinkal G, Ansieau S and Puisieux A: Generation of breast cancer stem cells through epithelial-mesenchymal transition. PLoS One 3: e2888, 2008.

35. Bjerkvig R, Johansson M, Miletic H and Niclou SP: Cancer stem cells and angiogenesis. Semin Cancer Biol 19: 279-284, 2009.

36. Dong J, Zhao Y, Huang Q, Fei X, Diao Y, Shen Y, Xiao H, Zhang T, Lan $Q$ and Gu X: Glioma stem/progenitor cells contribute to neovascularization via transdifferentiation. Stem Cell Rev 7: 141-152, 2011.

37. Monzani E and La Porta CA: Targeting cancer stem cells to modulate alternative vascularization mechanisms. Stem Cell Rev 4: 51-56, 2008

38. Sun D, Sun B, Liu T, Zhao X, Che N, Gu Q, Dong X, Yao Z, Li R, Li J, et al: Slug promoted vasculogenic mimicry in hepatocellular carcinoma. J Cell Mol Med 17: 1038-1047, 2013. 